TITLE:

\title{
A theoretical approach to La L(1)- edge XANES spectra of La complex oxides and their local configuration.
}

\section{$\operatorname{AUTHOR}(S)$ :}

Asakura, Hiroyuki; Shishido, Tetsuya; Teramura, Kentaro; Tanaka, Tsunehiro

\section{CITATION:}

Asakura, Hiroyuki ...[et al]. A theoretical approach to La L(1)-edge XANES spectra of La complex oxides and their local configuration.. The Journal of chemical physics 2015, 142(16): 164507.

\section{ISSUE DATE:}

2015-04-28

URL:

http://hdl.handle.net/2433/200902

\section{RIGHT:}

(c) 2015 American Institute of Physics. This article may be downloaded for personal use only. Any other use requires prior permission of the author and the American Institute of Physics. 


\section{AIP | The Journal of}

\section{A theoretical approach to La L1-edge XANES spectra of La complex oxides and their local configuration}

Hiroyuki Asakura, Tetsuya Shishido, Kentaro Teramura, and Tsunehiro Tanaka

Citation: The Journal of Chemical Physics 142, 164507 (2015); doi: 10.1063/1.4919055

View online: http://dx.doi.org/10.1063/1.4919055

View Table of Contents: http://scitation.aip.org/content/aip/journal/jcp/142/16?ver=pdfcov

Published by the AIP Publishing

\section{Articles you may be interested in}

Local order and electronic structure of $\mathrm{Pb} 1-\mathrm{xLaxZr0.40Ti0.6003}$ materials and its relation with ferroelectric properties

J. Appl. Phys. 111, 104110 (2012); 10.1063/1.4720472

High temperature oxygen near edge x-ray absorption fine structure valence band spectra and conductivity of LaFe3/4Ni1/4O3 from 300 to $773 \mathrm{~K}$

Appl. Phys. Lett. 99, 202112 (2011); 10.1063/1.3662970

Near-edge x-ray absorption and natural circular dichroism spectra of L-alanine: A theoretical study based on the complex polarization propagator approach

J. Chem. Phys. 127, 165104 (2007); 10.1063/1.2800024

Experimental and Calculated Ti K-Edge XANES Spectra of Pb1-xLaxTiO3 Ferroelectric Ceramic Compounds AIP Conf. Proc. 882, 496 (2007); 10.1063/1.2644571

Mg K-Edge XANES Spectra in Crystals and Oxide Glasses: Experimental vs. Theoretical Approaches AIP Conf. Proc. 882, 226 (2007); 10.1063/1.2644482

\section{AIP $\mid$ APL Photonics}

APL Photonics is pleased to announce Benjamin Eggleton as its Editor-in-Chief

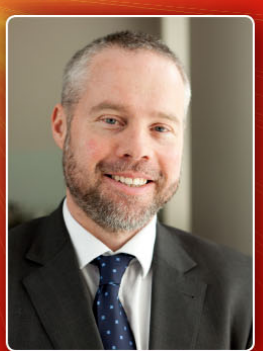




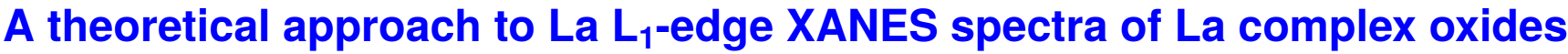 and their local configuration
}

\author{
Hiroyuki Asakura, ${ }^{1, a), b)}$ Tetsuya Shishido, ${ }^{2,3, b)}$ Kentaro Teramura, ${ }^{3,4,5, b)}$ \\ and Tsunehiro Tanaka ${ }^{3,4, a), b)}$ \\ ${ }^{1}$ Synchrotron Radiation Research Center, Nagoya University, Furo-cho, Chikusa, Nagoya 464-8603, Japan \\ ${ }^{2}$ Department of Applied Chemistry, Graduate School of Urban Environmental Sciences, Tokyo Metropolitan \\ University, 1-1 Minami-Osawa, Hachioji, Tokyo 192-0397, Japan \\ ${ }^{3}$ Elements Strategy Initiative for Catalysts and Batteries (ESICB), Kyoto University, Kyoto 615-8520, Japan \\ ${ }^{4}$ Department of Molecular Engineering, Graduate School of Engineering, Kyoto University, \\ Kyoto 615-8510, Japan \\ ${ }^{5}$ Precursory Research for Embryonic Science and Technology (PRESTO), Japan Science and Technology \\ Agency (JST), 4-1-8 Honcho, Kawaguchi, Saitama 332-0012, Japan
}

(Received 1 December 2014; accepted 14 April 2015; published online 27 April 2015)

\begin{abstract}
The characteristic peaks (pre-edge peaks) in the $\mathrm{La}_{1}$-edge $\mathrm{X}$-ray absorption near edge structure (XANES) of various La complex oxides were analyzed by both experimental and theoretical approaches. The pre-edge peak areas are correlated with the bond angle analysis (BAA) parameter, which we proposed as an indicator of the centrosymmetry or disorder of the local configuration of the La site. The origin of the pre-edge peak and the parameterization criteria of the BAA parameter were evaluated using theoretical calculations based on molecular orbital theory and multiple scattering theory. The theoretical calculations showed that the origin of the pre-edge peak at the $\mathrm{La}_{1}$-edge is electric dipole transition from $2 \mathrm{~s}$ to unoccupied states generated by $\mathrm{p}-\mathrm{d}$ hybridization. Medium-scale theoretical simulations of the $\mathrm{La} \mathrm{L}_{1}$-edge XANES spectra of thousands of virtual La aqueous complex models verified that the parameterization criteria of the BAA parameter are applicable to local configuration analysis of La. @ 2015 AIP Publishing LLC. [http://dx.doi.org/10.1063/1.4919055]
\end{abstract}

\section{INTRODUCTION}

X-ray absorption spectroscopy (XAS) is an important tool in science and technology and can be characterized by element specificity and penetration power. These features have realized a large number of applications for the element specific analysis of various materials, such as dopants or modifiers in dielectric materials,,${ }^{1,2}$ semi-conductors,,${ }^{3,4}$ glasses,${ }^{5-8}$ and catalysts. ${ }^{9}$ An $\mathrm{X}$-ray absorption spectrum is usually divided into two regions: the X-ray absorption near edge structure (XANES) which contains electronic and geometric information such as an oxidation number and centrosymmetry of the X-ray absorbing atom and the extended X-ray absorption fine structure (EXAFS) which contains important information about the local structure, such as the atomic distance between an X-ray absorbing atom and adjacent atoms and the coordination number of the neighboring atoms of the X-ray absorbing atom.

Another important feature of XAS is its applicability to amorphous materials. Powder X-ray diffraction (XRD) is a very useful technique that enables characterization of the geometric and electronic structures of crystalline well-ordered materials, and it is much more commonly used than XAS. In contrast, XAS can be applied to analyze the geometrical and chemical properties of an X-ray absorbing atom in disordered

\footnotetext{
a) Authors to whom correspondence should be addressed. Electronic addresses: h.asakura@nusr.nagoya-u.ac.jp and tanakat@moleng.kyoto-u.ac.

b) H. Asakura, T. Shishido, K. Teramura, and T. Tanaka contributed equally to this work.
}

materials, such as glasses and catalysts. For example, Marcus and Polman reported the difference in the local structure of $\mathrm{Er}$ cations in silica and sodium silicate glasses using $\mathrm{Er} \mathrm{L}_{3}$-edge EXAFS spectra. ${ }^{5}$ Antonio et al. applied EXAFS spectroscopy to Er- and Lu-doped sodium silicate glasses ${ }^{6}$ to investigate the local environments of $\mathrm{Er}$ and $\mathrm{Lu}$ atoms. They also used the $\mathrm{Er}$ and $\mathrm{Lu} \mathrm{L}_{3}$-edge XANES spectra and their second derivatives to obtain geometric information around the X-ray absorbing atoms. It is noteworthy that curve fitting analysis, which is the most common approach used for EXAFS spectra and is based on the EXAFS equation proposed by Sayer, Stern, and Lytle, ${ }^{10}$ is highly dependent on the initial structure model, which is usually difficult to construct for amorphous materials. EXAFS oscillation is the sum of discrete sine waves caused by interference between the photoelectron wave ejected from the X-ray absorbing atom and the potential of the neighboring atoms, whereas an XANES spectrum is a convolution spectrum of each XANES of every X-ray absorbing atom. Thus, we do not need a detailed structure model before interpreting the XANES spectrum, although the character of the local structure should be kept in mind, such as the electronic deficiency and local symmetry.

The relationship between XANES spectra and the geometric and electronic structures of various transition metal elements has been already investigated in detail. There are also several research papers on the XANES spectra of a series of lanthanide elements. For example, Mountjoy et al. ${ }^{8}$ reported the $\mathrm{L}_{1}$-edge XANES spectra of $\mathrm{Ce}, \mathrm{Nd}$, and Eu phosphate glasses and crystals and ascribed the presence of the small 
pre-edge shoulder peaks to $\mathrm{p}-\mathrm{d}$ hybridization, which depends on the centrosymmetry, analogous to the $\mathrm{La}_{1}$-edge XANES of $\mathrm{La}$ species in $\mathrm{K}_{2} \mathrm{O}-\mathrm{SiO}_{2}-\mathrm{La}_{2} \mathrm{O}_{3}$ glasses reported by Larson et al. ${ }^{11} \mathrm{D}$ ' Angelo et al. ${ }^{12-20}$ reported a series of $\mathrm{K}$ - and $\mathrm{L}_{3}$-edge XANES and EXAFS analyses of various lanthanide aqueous complexes. In particular, they used molecular dynamics to interpret the $\mathrm{Ln}_{\mathrm{L}_{3}}$-edge XANES spectra and obtained structural parameters such as the atomic distances between Ln atoms and adjacent $\mathrm{O}$ or other (e.g., $\mathrm{S}$ of solvent molecule) atoms with plausible solvation models. However, to the best of our knowledge, fundamental and systematic study focusing on the L-edge XANES spectra of one lanthanide element is relatively rare. This might be because the Ln L-edge XANES usually exhibits a monotonous featureless spectrum. In a pre-

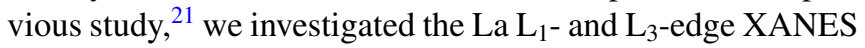
spectra of various La complex oxides and found correlations among the small pre-edge shoulder peak area observed in the La $\mathrm{L}_{1}$-edge XANES spectra, the full-width at half-maximum (FWHM) of the white line in the $\mathrm{La} \mathrm{L}_{3}$-edge XANES spectra, and the local structure of the La site. In addition, we also found a structural-spectral relationship between the $\mathrm{Ln} \mathrm{L}_{1}$ - and $\mathrm{L}_{3}$-edge XANES spectra of Pr, Sm, Nd, ${ }^{22} \mathrm{Eu}$, and $\mathrm{Gd} .{ }^{23}$ These results suggest that empirical quantitative analysis on the $\mathrm{Ln}$ $\mathrm{L}_{1^{-}}$and $\mathrm{L}_{3}$-edge XANES spectra could be a complementary method to conventional EXAFS curve fitting analysis.

In this paper, we discuss two issues for the possible application of Ln L-edge XANES measurement to the local structure analysis of Ln species. (1) The feasibility of the state-of-the-art theoretical simulation codes $\mathrm{ORCA}^{24}$ based on molecular orbital theory and $\mathrm{FEFF}^{25-27}$ based on multiple scattering theory to simulate the pre-edge shoulder peak in the experimental $\mathrm{La} \mathrm{L}_{1}$-edge XANES of reference La compounds. Ground state density functional theory (DFT) and time-dependent DFT (TD-DFT) calculations have already been used to interpret $\mathrm{C} \mathrm{K}$-edge, ${ }^{28} \mathrm{~S}$ K-edge, ${ }^{29} \mathrm{Cl} \mathrm{K}$-edge, ${ }^{30}$ and $\mathrm{Mn} \mathrm{K}$-edge ${ }^{31}$ XANES spectra. However, the application of theoretical simulations to L-edge XANES spectroscopy of heavy elements is rare. The origin of the peak is also discussed. (2) By medium-scale simulations of the pre-edge peak in the $\mathrm{La} \mathrm{L}_{1}$-edge XANES spectra for thousands of virtual La aqueous complex models with ORCA and FEFF, we propose and discuss the validity of parameterization criteria of the local configuration bond angle analysis (BAA) parameter, which is defined such that all of the independent angles consist of a $\mathrm{La}$ atom and two adjacent $\mathrm{O}$ atoms.

\section{MATERIALS AND METHODS}

\section{A. Sample preparation of lanthanum oxides}

The lanthanum complex oxides, $\mathrm{La}_{4} \mathrm{PdO}_{7}, \mathrm{La}_{2} \mathrm{CuO}_{4}, \mathrm{La}$ $\mathrm{CuSrO}_{4}, \mathrm{LaFeSrO}_{4}, \mathrm{LaSrAlO}_{4}, \mathrm{LaCoO}_{3}$, and $\mathrm{LaAlO}_{3}$, were prepared by solid-state reaction. $\mathrm{La}_{4} \mathrm{PdO}_{7}: \mathrm{La}_{2} \mathrm{O}_{3}(325 \mathrm{mg}$, $1.0 \mathrm{mmol})$ and $\mathrm{Pd}(\mathrm{OAc})_{2}(124 \mathrm{mg}, 0.5 \mathrm{mmol}) ; \mathrm{La}_{2} \mathrm{CuO}_{4}$ : $\mathrm{La}_{2} \mathrm{O}_{3}(652 \mathrm{mg}, 2.0 \mathrm{mmol})$ and $\mathrm{Cu}(\mathrm{OAc})_{2}(363 \mathrm{mg}, 2.0 \mathrm{mmol})$; $\mathrm{LaCuSrO}_{4}: \mathrm{La}_{2} \mathrm{O}_{3}(322 \mathrm{mg}, 1.0 \mathrm{mmol}), \mathrm{Cu}(\mathrm{OAc})_{2}(362 \mathrm{mg}$, $2.0 \mathrm{mmol}$ ), and $\mathrm{SrCO}_{3}(295 \mathrm{mg}, 2.0 \mathrm{mmol}) ; \mathrm{LaFeSrO}_{4}: \mathrm{La}_{2} \mathrm{O}_{3}$ (325 mg, $1.0 \mathrm{mmol}), \mathrm{Fe}\left(\mathrm{NO}_{3}\right)_{3} \cdot 9 \mathrm{H}_{2} \mathrm{O}(810 \mathrm{mg}, 2.0 \mathrm{mmol})$, and $\mathrm{SrCO}_{3}(300 \mathrm{mg}, 2.0 \mathrm{mmol}) ; \mathrm{LaSrAlO}_{4}: \mathrm{La}_{2} \mathrm{O}_{3}(328 \mathrm{mg}$,
$1.0 \mathrm{mmol}), \mathrm{SrCO}_{3}(310 \mathrm{mg}, 2.0 \mathrm{mmol})$, and $\mathrm{Al}(\mathrm{NO})_{3} \cdot 6 \mathrm{H}_{2} \mathrm{O}$ (750 mg, $2.0 \mathrm{mmol}) ; \mathrm{LaCoO}_{3}: \mathrm{La}_{2} \mathrm{O}_{3}(327 \mathrm{mg}, 1.0 \mathrm{mmol})$ and $\mathrm{Co}\left(\mathrm{NO}_{3}\right)_{2} \cdot 6 \mathrm{H}_{2} \mathrm{O}(614 \mathrm{mg}, 2.0 \mathrm{mmol})$; and $\mathrm{LaAlO}_{3}: \mathrm{La}_{2} \mathrm{O}_{3}$ (325 mg, $1.0 \mathrm{mmol})$ and $\mathrm{Al}\left(\mathrm{NO}_{3}\right)_{3} \cdot 6 \mathrm{H}_{2} \mathrm{O}(764 \mathrm{mg}, 2.0 \mathrm{mmol})$. These mixtures were placed in alumina crucibles and heated at $1423 \mathrm{~K}$ for $24 \mathrm{~h}$. The identification was performed by comparison of the XRD patterns of the prepared samples and previously reported structures (Figure $\mathrm{S}^{50}$ ).

\section{B. XAS measurements and data reduction}

The La $\mathrm{L}_{1}$-edge XANES spectra of these materials were measured at the BL5S1 hard X-ray XAFS beamline at the Aichi Synchrotron Radiation Center ${ }^{32}$ (AichiSR; Aichi Science and Technology Foundation, Aichi, Japan). The XANES spectra of the powder samples were recorded in transmission mode under ambient conditions using a $\mathrm{Si}(111)$ double crystal monochromator. The photon energy was calibrated to the preedge peak $(4966.0 \mathrm{eV})$ observed in the Ti K-edge XANES spectrum of Ti foil. The powder samples were mixed with an appropriate amount of boron nitride and pressed into pellets. The incident and transmitted $\mathrm{X}$-ray fluxes were measured with ion chambers filled with $\mathrm{He}(70 \%) / \mathrm{N}_{2}(30 \%)$ and $\mathrm{N}_{2}(15 \%) / \mathrm{Ar}$ $(85 \%)$. Higher harmonic $\mathrm{X}$-rays were cutoff with a proper glancing angle of Rh-coated collimating and focusing mirrors. A typical data reduction procedure (e.g., background removal or normalization) was carried out with Athena (version 0.9.18) included in the Demeter package. ${ }^{33}$ Curve fitting analysis of the $\mathrm{La} \mathrm{L}_{1}$-edge XANES spectra was also performed with the peak fitting function implemented in Athena and the multipeak fitting procedure implemented in Igor Pro (version 6.35, Wavemetrics, Lake Oswego, OR, USA).

\section{Theoretical calculations}

Theoretical simulations of the $\mathrm{La}_{1}$-edge XANES spectra for various reference samples were performed with the restricted open-shell configuration interaction single-excitation (ROCIS) method ${ }^{34-37}$ by DFT implemented in the ORCA package. ${ }^{24}$ The geometry optimization and ROCIS calculation were performed using the B3LYP functional with Ahlrich's polarized def2-TZVP split valence basis sets ${ }^{38,39}$ of triple $\zeta$ quality for all of the atoms in combination with the auxiliary basis set def2-TZVP/J for the resolution of identity. The DFT/ROCIS calculations were performed with the converged restricted Kohn-Sham wavefunctions. Scalar relativistic effects were explicitly incorporated by the zeroth order regular approximation (ZORA) correction. ${ }^{40}$

Instead of a large cluster model of the lanthanide complex oxides, we used virtual aqueous complex models which are virtual minimal $\mathrm{La}$ aqueous complexes with the exact relative positions of $\mathrm{La}$ and $\mathrm{O}$ atoms taken from the X-ray crystal structure of the corresponding La complex oxide in a similar manner to the previous literature. ${ }^{35}$ For example, an aqueous complex model for $\mathrm{LaCoO}_{3}$ was constructed and examined in the following procedure (Figure 1). First, we extracted the positions of $\mathrm{La}$ and the adjacent $12 \mathrm{O}$ atoms from the crystal structure reconstructed from the XRD pattern of $\mathrm{LaCoO}_{3}$, which matches that of prepared $\mathrm{LaCoO}_{3}$. Second, two $\mathrm{H}$ atoms were placed next to each $\mathrm{O}$ atom to terminate the $\mathrm{O}$ atoms and 


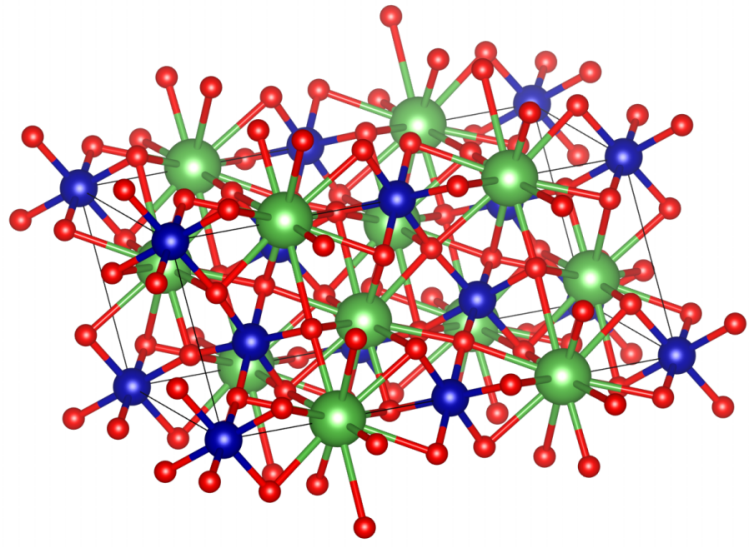

\author{
$\mathrm{LaO}_{12}$ moiety \\ from Crystal Structure
}

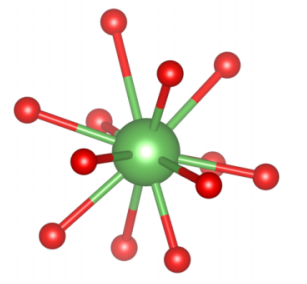

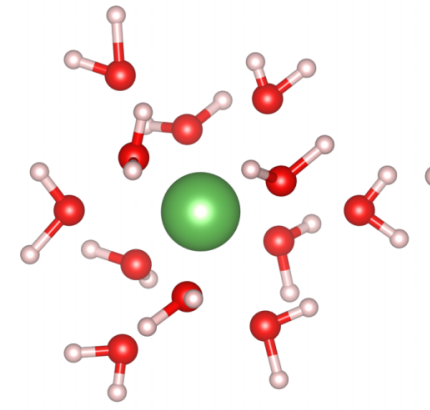

$\mathrm{La}\left(\mathrm{H}_{2} \mathrm{O}\right)_{12}$ model

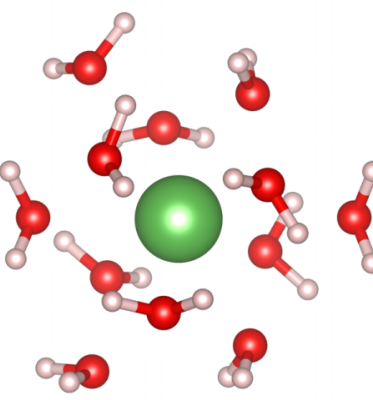

$\mathrm{La}\left(\mathrm{H}_{2} \mathrm{O}\right)_{12}$ optimized by MD

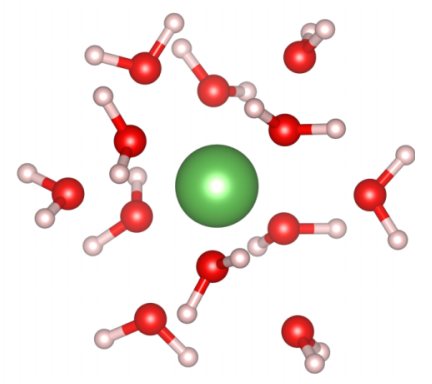

$\mathrm{La}\left(\mathrm{H}_{2} \mathrm{O}\right)_{12}$ optimized by DFT
FIG. 1. Example of an aqueous model complex of $\mathrm{La}\left(\mathrm{H}_{2} \mathrm{O}\right)_{12}$ from $\mathrm{LaCoO}_{3}$. compensate the negative charges, and their positions were optimized in the molecular dynamics framework with the universal force field ${ }^{41}$ keeping the positions of La and $\mathrm{O}$ atoms fixed using Avogadro, ${ }^{42}$ an advanced semantic chemical editor. Finally, the positions of the $\mathrm{H}$ atoms were optimized again under the same condition as the DFT/ROCIS calculation described above.

Because we are considering lanthanide elements, spin orbit coupling (SOC) could have a significant effect on the simulated spectra. Thus, we performed $\mathrm{La} \mathrm{L}_{1}$-edge XANES simulations with and without the SOC effect by turning the "SOC" option in the ROCIS directive of ORCA on and off. We found that the simulated spectra were almost the same for the present La models with and without the SOC effect. Thus, the SOC effect on the $\mathrm{La} \mathrm{L}_{1}$-edge XANES is negligible in our case. In addition, the effect of higher multiplicity states ("DoHigher true" option in ROCIS directive in the input file for ORCA) was included in the present simulations.

XANES and local density of states' (LDOSs) calculations of the La complex oxide cluster models were also performed with FEFF. The cluster models were constructed from the crystal structure with the ATOMS package, ${ }^{43}$ which contains all atoms within $10 \AA$ of the target X-ray absorbing atom (La). If there were nonequivalent La sites in the structure, a structural model was constructed for each different La site. We took into account not only the electric dipole transition but also the electric quadrupole transition for the XANES simulation using the Hedin-Lundqvist function for the fine structure and the ground state function for the background function with self- consistent field and full multiple scattering (FMS) calculations. For the LDOS calculation, the Lorentzian broadening parameter was $1.0 \mathrm{eV}$. These calculations were performed on the supercomputer located at the Information Technology Center, Nagoya University.

\section{RESULTS AND DISCUSSION}

\section{A. La L1-edge XANES of La complex oxides}

Figure 2 shows the $\mathrm{La} \mathrm{L}_{1}$-edge XANES spectra of $\mathrm{La}_{4} \mathrm{Pd}$ $\mathrm{O}_{7}, \mathrm{La}_{2} \mathrm{CuO}_{4}, \mathrm{LaCuSrO}_{4}, \mathrm{LaFeSrO}_{4}, \mathrm{LaSrAlO}_{4}, \mathrm{LaCoO}_{3}$, and $\mathrm{LaAlO}_{3}$, which are structures with La surrounded by several $\mathrm{O}$ atoms with various positions relative to $\mathrm{La}$. The overall structures of the $\mathrm{L}_{1}$-edge XANES spectra, such as the absorption edge of each compound, are similar, which indicates that La in each compound is trivalent (i.e., $\mathrm{La}^{3+}$ : $[\mathrm{Xe}] 5 \mathrm{~d}^{0} 6 \mathrm{~s}^{0} 6 \mathrm{p}^{0}$ ). However, the strength of the characteristic shoulder peak observed at about $6275 \mathrm{eV}$ varied among the samples. Larson et al. ${ }^{11}$ assumed that the origin of the shoulder peaks in the $\mathrm{La} \mathrm{L}_{1}$-edge XANES spectra was electric quadrupole transition from $2 \mathrm{~s}$ to $5 \mathrm{~d}$ unoccupied states. Because it is the $\mathrm{La} \mathrm{L}_{1}$-edge XANES spectrum, the origin of the transition can be an electric dipole transition from the $\mathrm{s}$ to the $\mathrm{p}$ state or an electric quadrupole transition from $s$ to $d$, but not a transition from $s$ to $s$, which is known as the selection rule. The spectra of $\mathrm{LaAlO}_{3}$ and $\mathrm{LaCoO}_{3}$ (12 $\mathrm{O}$ atoms around each $\mathrm{La}$ atom) have the smallest shoulder peaks. The spectra of $\mathrm{LaSrAlO}_{4}, \mathrm{LaSrFeO}_{4}$, $\mathrm{LaSrCuO}_{4}$, and $\mathrm{La}_{2} \mathrm{CuO}_{4}$ (nine $\mathrm{O}$ atoms around each $\mathrm{La}$ atom) 


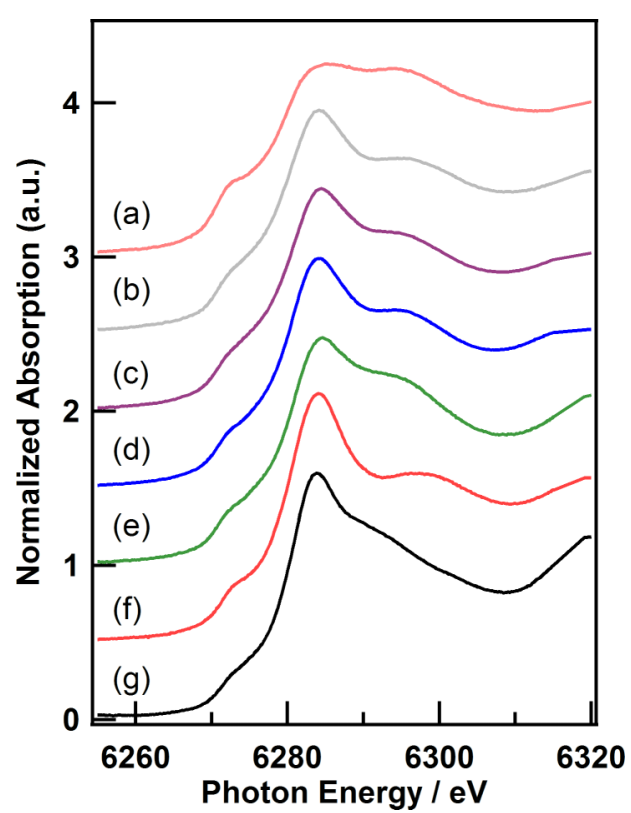

FIG. 2. La $\mathrm{L}_{1}$-edge XANES spectra of La complex oxides: (a) $\mathrm{La}_{4} \mathrm{PdO}_{7}$, (b) $\mathrm{La}_{2} \mathrm{CuO}_{4}$, (c) $\mathrm{LaCuSrO}_{4}$, (d) $\mathrm{LaFeSrO}_{4}$, (e) $\mathrm{LaSrAlO}_{4}$, (f) $\mathrm{LaCoO}_{3}$, and (g) $\mathrm{LaAlO}_{3}$. Reprinted with permission from Asakura et al., Inorg. Chem. 53(12), 6048-6053 (2014). Copyright 2014 American Chemical Society.

have more evident shoulder peaks. Furthermore, the spectrum of $\mathrm{La}_{4} \mathrm{PdO}_{7}$ (seven $\mathrm{O}$ atoms around each $\mathrm{La}$ atom) has the largest shoulder peak among the samples. The crystal structure of each La compound is shown in Figure S2. ${ }^{50}$ In our previous study ${ }^{21}$ we suggested that there is a correlation between the shoulder peak intensity in the La $\mathrm{L}_{1}$-edge XANES spectra and the local structure of the La atoms of La complex oxides through the primitive parameterization of the degree of local centrosymmetry or complexity (BAA parameter), based on all of the angles of every two adjacent $\mathrm{O}$ atoms to La. The BAA parameter is defined as

$$
\mathrm{BAA}=\frac{1}{n} \sum\left|\cos \left(\theta_{\mathrm{ijk}}\right)\right|,
$$

where $n$ is the number of independent angles and $\theta_{\mathrm{ijk}}$ is the angle formed by the central atom $\mathrm{j}$ (i.e., La) and two of its adjacent atoms $\mathrm{i}$ and $\mathrm{k}$ (i.e., two $\mathrm{O}$ atoms).

Figure 3 shows the dependence of the pre-edge peak area of the $\mathrm{La} \mathrm{L}_{1}$-edge XANES spectra on the BAA parameter for $\mathrm{La}_{4} \mathrm{PdO}_{7}, \mathrm{La}_{2} \mathrm{CuO}_{4}, \mathrm{LaCuSrO}_{4}, \mathrm{LaFeSrO}_{4}, \mathrm{LaSrAlO}_{4}$, $\mathrm{LaCoO}_{3}$, and $\mathrm{LaAlO}_{3}$. The correlation indicates the possibility

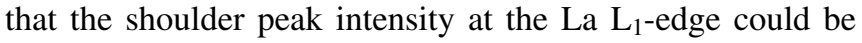
an indicator of the local configuration of La. This result encouraged us to extend the well-known analysis strategy for the pre-edge peak of the K-edge XANES spectra of 3d early transition metals to the $\mathrm{Ln}_{\mathrm{L}}$-edge XANES spectra. The BAA parameter was inspired by the discussion in metallurgy to interpret the local structure of a metal atom in an ill-defined model generated by molecular dynamics ${ }^{44}$ and might have a relationship with the pre-edge peak intensity induced by the $\mathrm{p}-\mathrm{d}$ hybridization. However, the parameterization criteria of the BAA parameter appear to be too primitive to associate it with a physical meaning. Thus, we used another approach to validate the BAA parameter as an indicator of the local

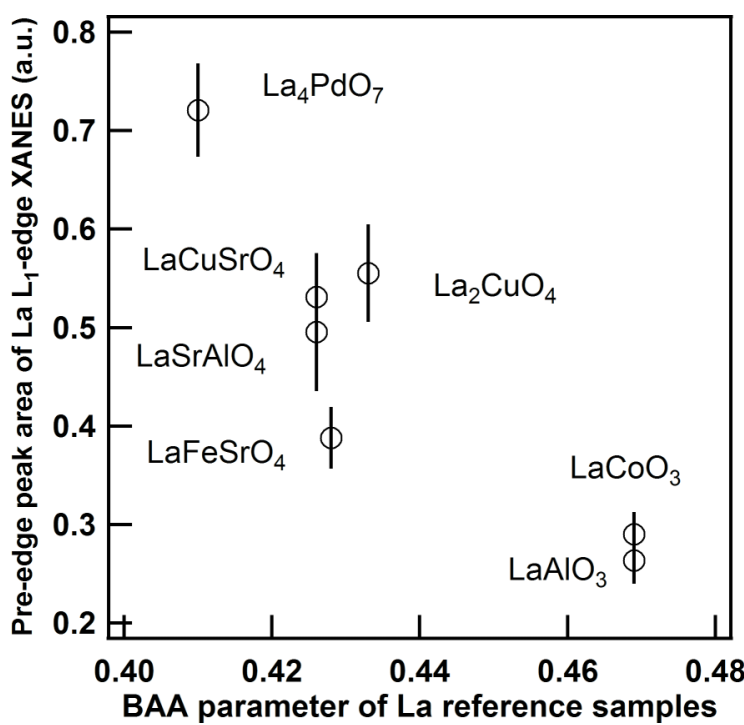

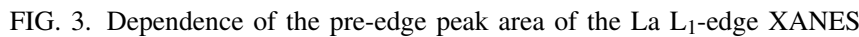
spectrum on the BAA parameter for $\mathrm{La}_{4} \mathrm{PdO}_{7}, \mathrm{La}_{2} \mathrm{CuO}_{4}, \mathrm{LaCuSrO}_{4}$, $\mathrm{LaFeSrO}_{4}, \mathrm{LaSrAlO}_{4}, \mathrm{LaCoO}_{3}$, and $\mathrm{LaAlO}_{3}$. Reprinted with permission from Asakura et al., Inorg. Chem. 53(12), 6048-6053 (2014). Copyright 2014 American Chemical Society.

symmetry by $\mathrm{La}_{1}$-edge XANES simulation of medium-scale (up to thousands of) virtual $\mathrm{La}\left(\mathrm{H}_{2} \mathrm{O}\right)_{n}$ complex models.

Before the medium-scale simulations, we first attempted to reproduce the pre-edge peak feature at the $\mathrm{La} \mathrm{L}_{1}$-edge of the reference samples with the ORCA package based on TD-DFT/ROCIS and the FEFF package based on multiple scattering theory.

\section{B. DFT/ROCIS, TD-DFT, and FMS calculations of the La $\mathrm{L}_{1}$-edge XANES of La complex oxides}

Figure 4(a) shows the experimental $\mathrm{La} \mathrm{L}_{1}$-edge XANES spectrum of $\mathrm{La}_{4} \mathrm{PdO}_{7}$ and the corresponding simulated spectrum of the $\mathrm{La}\left(\mathrm{H}_{2} \mathrm{O}\right)_{7}$ complex model from the DFT/ROCIS calculation. The simulated spectrum was broadened by the Gaussian function by $4 \mathrm{eV}$ (FWHM) and shifted toward higher energy by $19 \mathrm{eV}$. Each transition line of $\mathrm{LaAlO}_{3}$ without broadening is shown in Figure S3. ${ }^{50}$ The peak positions of the simulated spectrum at about 6275,6284 , and $6288 \mathrm{eV}$ agree reasonably well with the three peak positions of the experimental spectrum at about 6273, 6284, and $6292 \mathrm{eV}$. Possible reasons for the slight difference between the peak positions of the simulated and experimental spectra are the transition to the vacuum level was not included in the present conditions, the line width broadening applied to each transition should not be same to reproduce the experimental spectrum, and no multiple scattering effect was considered, which can potentially change the fine structure. The simulated $\mathrm{La} \mathrm{L}_{1}$-edge XANES spectra of other virtual $\mathrm{La}$ aqueous model complexes also reproduced the corresponding La complex oxide spectra (Figure 4), and the shoulder peaks of the experimental spectra are clearly reproduced as the lowest peaks of the simulated spectra. In addition, TD-DFT calculations of the same models were performed with similar parameters, and they also reproduced the main three peaks observed from 6270 to $6300 \mathrm{eV}$ (Figure $\mathrm{S}^{50}{ }^{50}$ ). 

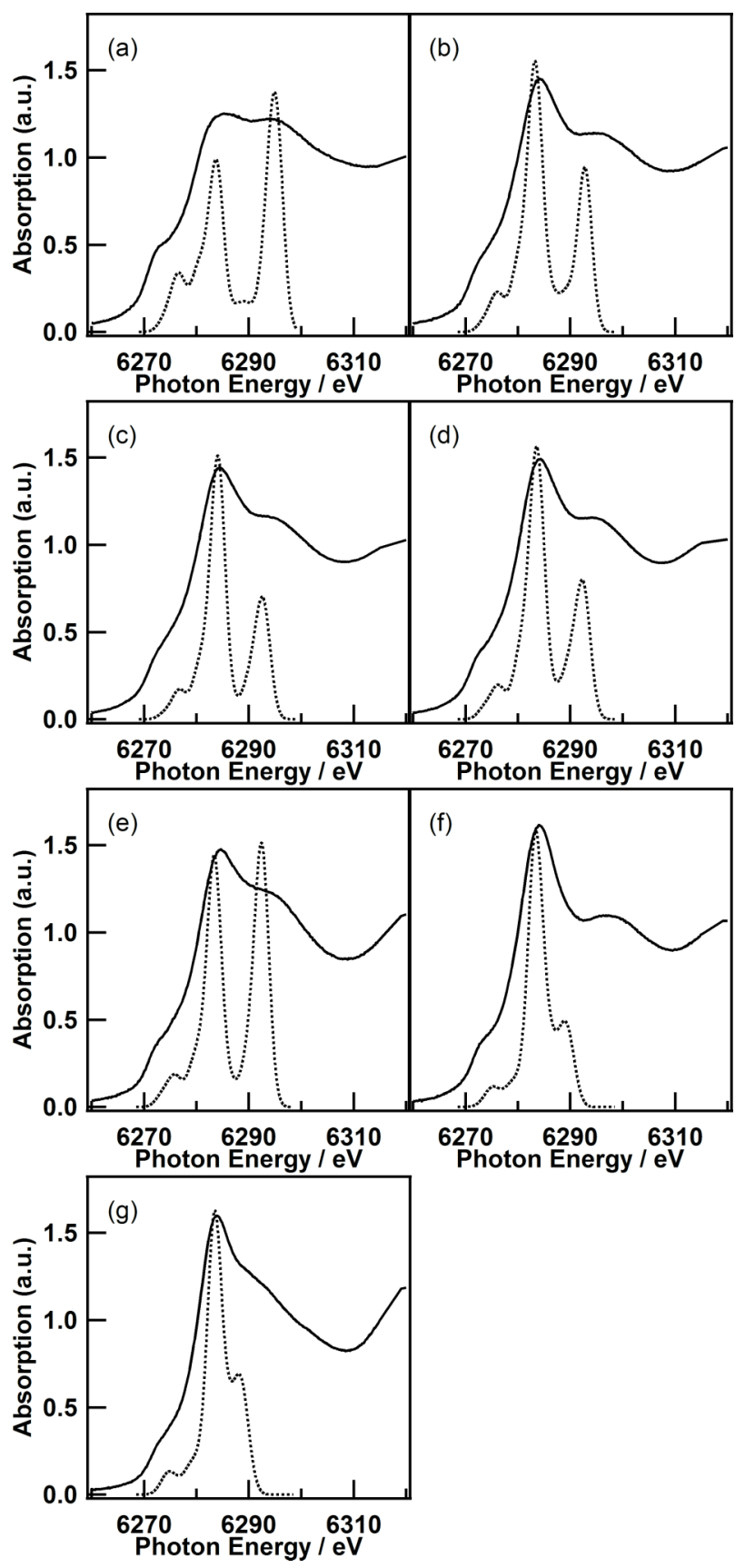

FIG. 4. Experimental and DFT/ROCIS simulated $\mathrm{La} \mathrm{L}_{1}$-edge XANES spectra of various $\mathrm{La}$ compounds and $\mathrm{La}\left(\mathrm{H}_{2} \mathrm{O}\right)_{n}$ model complexes: (a) $\mathrm{La}_{4} \mathrm{PdO}_{7}$, (b) $\mathrm{La}_{2} \mathrm{CuO}_{4}$, (c) $\mathrm{LaCuSrO}_{4}$, (d) $\mathrm{LaFeSrO}_{4}$, (e) $\mathrm{LaSrAlO}_{4}$, (f) $\mathrm{LaCoO}_{3}$, and (g) $\mathrm{LaAlO}_{3}$ (experimental: solid line and simulated: dotted line).

For quantitative analysis of the shoulder peak simulated with ORCA, we compared the first prominent peak area integrated from 6270 to $6278 \mathrm{eV}$ with the BAA parameter. The BAA parameter has already been shown to be correlated with the experimental shoulder peak in the $\mathrm{La} \mathrm{L}_{1}$-edge XANES extracted by curve fitting analysis with a cubic function as a background and a Gaussian function (Figure 3). Figure 5 shows an almost inverse linear relationship between the lowest energy peak area and the BAA parameter. Thus, these results demonstrate that the DFT/ROCIS and TD-DFT calculations with ORCA are capable of simulating the $\mathrm{La}_{1}$-edge XANES spectra at least in the lower excited states region of various

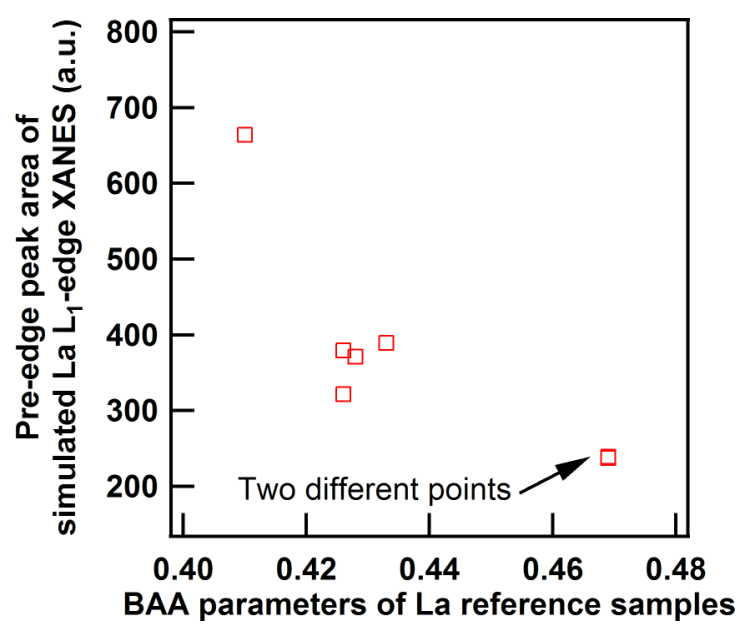

FIG. 5. Dependence of the peak area of the lowest energy peak of the simulated $\mathrm{La} \mathrm{L}_{1}$-edge XANES spectrum on the BAA parameter for La aqueous complex models of $\mathrm{La}_{4} \mathrm{PdO}_{7}, \mathrm{La}_{2} \mathrm{CuO}_{4}, \mathrm{LaCuSrO}_{4}, \mathrm{LaFeSrO}_{4}, \mathrm{LaSrAlO}_{4}$, $\mathrm{LaCoO}_{3}$, and $\mathrm{LaAlO}_{3}$.

real compounds, even though the structural model used for the DFT calculation was a very simple aqueous La complex. To verify the correlation, we performed XANES simulations of thousands of virtual La complex models, which will be discussed in Sec. III D.

Figure 6 shows the simulated $\mathrm{La} \mathrm{L}_{1}$-edge XANES spectra of the reference compounds with the FEFF code under the FMS framework including electric dipoles and quadrupole transitions. All of the energies are relative to the predicted Fermi energy for that compound. The FEFF calculations of the La complex oxide cluster models produced $\mathrm{La} \mathrm{L}_{1}$-edge XANES spectra comparable with the experimental spectra, except for $\mathrm{LaSrAlO}_{4}$, including the pre-edge shoulder peaks just above the Fermi level. The order of the spectra is the same as in Figure 2, although spectrum (e) for $\mathrm{LaSrAlO}_{4}$ is omitted because we could not produce a plausible spectrum for this

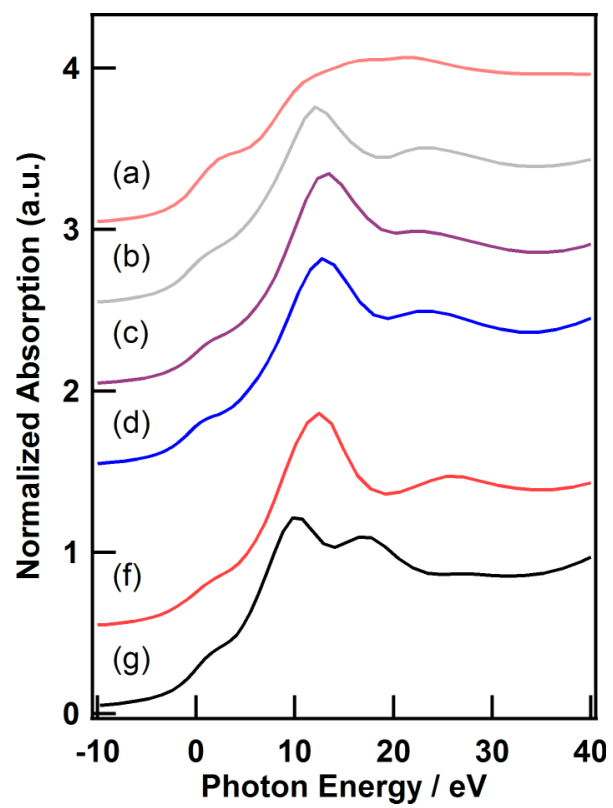

FIG. 6. Simulated $\mathrm{La}_{1}$-edge XANES spectra of various La complex oxide cluster models: (a) $\mathrm{La}_{4} \mathrm{PdO}_{7}$, (b) $\mathrm{La}_{2} \mathrm{CuO}_{4}$, (c) $\mathrm{LaCuSrO}_{4}$, (d) $\mathrm{LaFeSrO}_{4}$, (f) $\mathrm{LaCoO}_{3}$, and (g) $\mathrm{LaAlO}_{3}$. 
compound. The pre-edge peak area was also quantitatively evaluated in the same manner as the experimental areas, that is, curve fitting analysis with one background cubic function and one Gaussian function for the pre-edge peak. The peak area showed a clear correlation with the BAA parameter, in a manner similar to the results in Figure 5 (Figure $\mathrm{S} 5^{50}$ ). Thus, the FEFF code is also capable of quantitatively simulating the $\mathrm{La} \mathrm{L}_{1}$-edge XANES spectra. We also evaluated the contribution of the electric quadrupole transition to the pre-edge peak and found that the increase of the pre-edge peak area by the quadrupole transition was almost constant among the reference compounds. ${ }^{21}$ The magnetic dipole transition is another possible origin of the pre-edge peak. However, as Brouder discussed in his paper, ${ }^{45}$ the contribution is negligible even if the SOC effect is taken into account.

\section{Origin of the pre-edge peak at the La $\mathrm{L}_{1}$-edge XANES of various La compounds}

It is also important to clarify the origin of the shoulder peak in the $\mathrm{La}_{1}$-edge XANES spectrum. The shoulder peak in the Ln $\mathrm{L}_{1}$-edge XANES spectrum has been already discussed in several papers. In an XAS study of La coordination, Larson et al. ${ }^{11}$ reported that the shoulder peak in the $\mathrm{La} \mathrm{L}_{1}$-edge XANES spectra of $\mathrm{K}_{2} \mathrm{O}-\mathrm{SiO}_{2}-\mathrm{La}_{2} \mathrm{O}_{3}$ glasses is because of the $2 \mathrm{~s}$ to $\mathrm{d}$ transition, which may mean electric dipole transition to $\mathrm{p}-\mathrm{d}$ hybridized states or electric quadrupole transition to $\mathrm{d}$ components. Materlik et al. ${ }^{46}$ discussed the $\mathrm{Ce}, \mathrm{Sm}, \mathrm{Gd}$, and $\mathrm{Er}$ $\mathrm{L}_{1}$-edge XANES spectra in the atomic and condensed (solid or metallic) states and concluded that the shoulder peak is associated with the p-projected density of conduction-band states. Chaboy et al. ${ }^{47}$ explained the decrease of the shoulder peak intensity in the $\mathrm{Ce}$ and $\mathrm{La} \mathrm{L}_{1}$-edge XANES spectra of $\mathrm{CeFe}_{2}$, $\mathrm{CeRu}_{2}$, and $\mathrm{LaRu}_{2}$ after hydrogenation by $\mathrm{p}-\mathrm{d}$ hybridization. Ishimatsu et al. ${ }^{48}$ explained the disappearance of the shoulder peak in the $\mathrm{La} \mathrm{L}_{1}$-edge spectrum with hydrogenation of $\mathrm{La}$ metal in the same way. The pre-edge peak has been already discussed for Ln compounds with well-defined structures from the viewpoint of the $p$ component of the unoccupied states. However, to the best of our knowledge, the shoulder peak and $p$ orbital hybridization of La have not been systematically discussed for various coordination environments of $\mathrm{La}$.

A possible way to evaluate the $\mathrm{p}$ component contribution to the unoccupied state is population analysis of the virtual orbitals of the ground-state model. For example, George et al. ${ }^{49}$ discussed the pre-edge peak observed in the Ti K-edge XANES spectra of $\mathrm{TiCl}_{4}, \mathrm{TiCpCl}_{3}$, and $\mathrm{TiCp}_{2} \mathrm{Cl}_{2}(\mathrm{Cp}=$ cyclopentadienyl) by ground-state DFT calculations with the Amsterdam density functional package from the viewpoint of $3 d-4 p$ mixing and metal-ligand covalency. They found that the change of the pre-edge peak area at the Ti K-edge can be clearly explained by the amount of the Ti $4 p$ component of the unoccupied orbitals. We also performed single-point calculations of the Ti complexes with the ORCA package and confirmed that the Löwdin population analysis agrees with results of George et al. However, this was not the case for our virtual La aqueous complex models. In contrast to the single-point calculation result of the tetrahedral $\mathrm{TiCl}_{4}$ complex that exhibits a clear picture of the ligand field splitting to $e$ and $t_{2}$ states, the ground-state calculations of the La aqueous model complexes $\mathrm{La}\left(\mathrm{H}_{2} \mathrm{O}\right)_{n}(n=7-12)$ produced complicated virtual orbitals, which are difficult to interpret with the experimental results. This indicates that virtual complex models and ground-state calculations are not adequate for pre-edge peak interpretation.

As discussed in a previous paper, ${ }^{21}$ calculation of the LDOS at the X-ray absorbing atom site with FEFF could be another way to clarify the origin of the pre-edge peak. An example of the LDOS at the excited La site and the corresponding $\mathrm{L}_{1}$-edge XANES spectrum of $\mathrm{LaAlO}_{3}$ are shown in Figure 7. The figure shows that the two main peaks at ca. 10 and $17 \mathrm{eV}$ above the Fermi energy can be explained by the p-projected DOS of the unoccupied states, and the pre-edge transition at about the Fermi energy consists of the unoccupied state with $p$ and d components. The calculated DOS of the other reference samples can also explain the main peaks in the $\mathrm{L}_{1}$ edge XANES spectra.

In addition, the amount of the $\mathrm{p}$ component in the preedge peak region of the La models is correlated with the BAA parameter, as shown in Figure 8. The FEFF results indicate that the origin of the pre-edge peak at the $\mathrm{La}_{1}$-edge can be assigned to the $2 \mathrm{~s}$ to $\mathrm{p}$ electric dipole transition induced by the perturbation of the $\mathrm{p}$ orbitals of the adjacent $\mathrm{O}$ atoms of coordinating ligands, as a result of a decrease of the symmetry by the intersection between the orthogonal $\mathrm{p}$ orbitals and unoccupied $\mathrm{d}$ orbitals. A schematic view of d orbital splitting of 7, 8, 9, and 12 coordinated species with relatively high symmetry such as pentagonal bipyramidal, cubic, tricapped trigonal prism is provided in Figure $\mathrm{S}^{50}{ }^{50}$ However, the $\mathrm{La} \mathrm{L}_{3}$-edge XANES spectra of these La compounds reported in our previous paper only exhibit very small difference and the fact indicates that the $d$ orbital broadening did not make a significant effect on the pre-edge feature in the $\mathrm{L}_{1}$-edge XANES region.

\section{DFT/ROCIS simulation of La $\mathrm{L}_{1}$-edge XANES for a number of La aqueous complex models and the relationship between the pre-edge peak

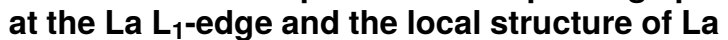

As discussed above, both the DFT/ROCIS and FMS calculations with ORCA or FEFF semi-quantitatively reproduced the characteristic features of the experimental data of the real La complex oxides, supporting that the BAA parameter can be an indicator of the local configuration of the La atoms. Unfortunately, there are two weak points: the validity of the BAA parameter definition and the relatively small number of reference samples. The former problem is not so easy. For example, the perfect pentagonal bipyramid geometry of a seven-coordinated moiety belongs to the $\mathrm{D}_{5 \mathrm{~h}}$ point group and its electronic states can be explained in a similar way to a fourcoordinated tetrahedral group or a six-coordinated octahedral group based on point group theory. However, the main aim of the present analysis method is to investigate atoms in amorphous materials, which have irregular configurations beyond the scope of point group theory. We then took a different approach to the problem, where a number of virtual $\mathrm{La}\left(\mathrm{H}_{2} \mathrm{O}\right)_{n}$ complex models were constructed with the La atom geometrically shifted from the original position. Defining the La atom of a virtual $\mathrm{La}$ aqueous complex model to be located at the origin 


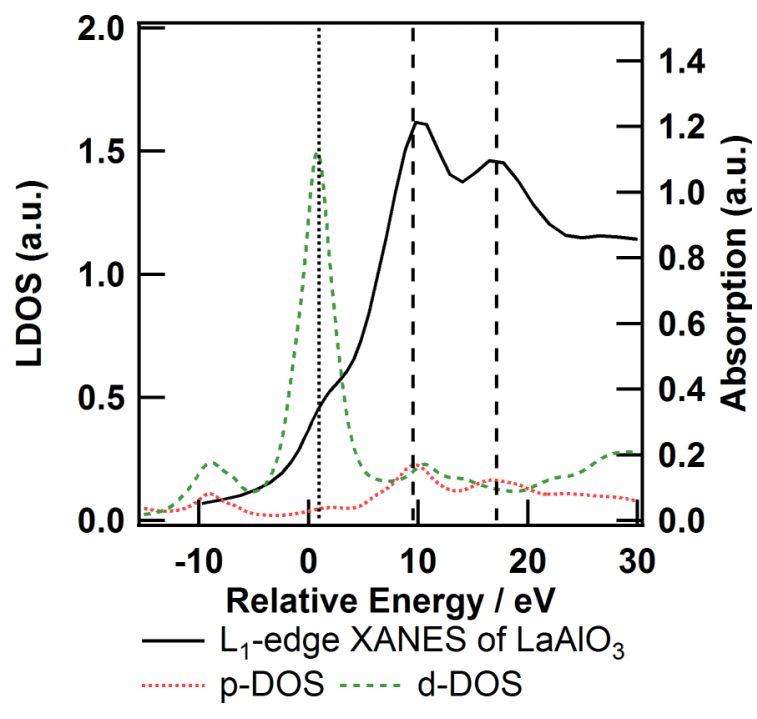

FIG. 7. $\mathrm{La} \mathrm{L}_{1}$ XANES spectrum and LDOS at the La site of $\mathrm{LaAlO}_{3}$ calculated with FEFF. The relative energy is with respect to the estimated Fermi energy.

$[(0,0,0)]$ in the Cartesian axis system, we virtually constructed a $0.40 \AA$ A cubic grid with a $0.05 \AA$ mesh, which has $9 \times 9 \times 9$ $=729$ intersection points including the origin. Then, to construct new complex models, the La atom was moved to each intersection point without moving the other atoms. Calculation and evaluation of the pre-edge peak in the $\mathrm{La}_{1}$-edge XANES spectra of these new complex models were performed in the same manner as the previous models.

Figure 9 shows the simulated results of a number of virtual $\mathrm{La}\left(\mathrm{H}_{2} \mathrm{O}\right)_{n}(n=7,8,9,10,12)$ complex models and the reference models already shown in Figure 5. Even though the parameterization criteria for the BAA parameter are still unclear from the viewpoint of the physical description, the result shows a correlation between the pre-edge peak area and the

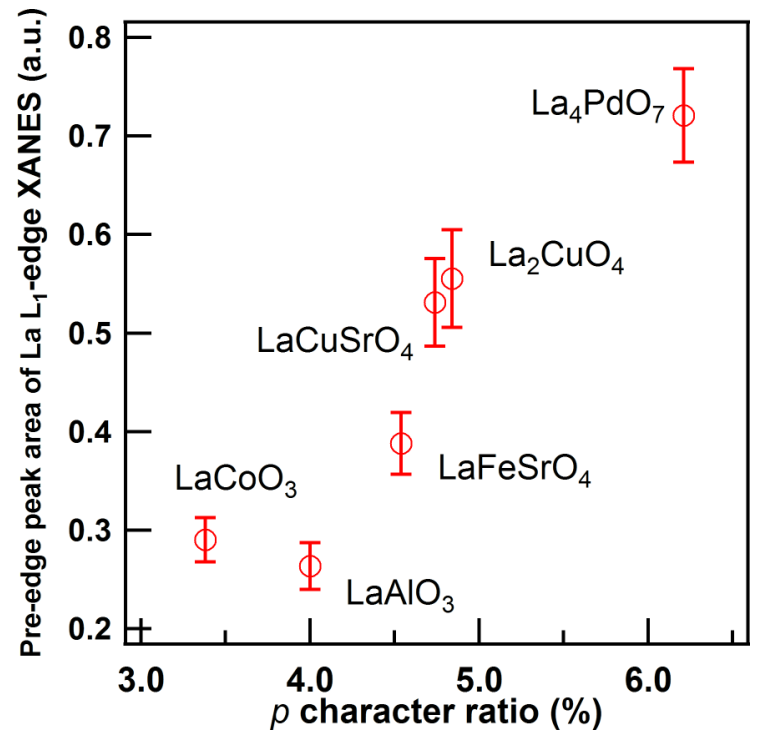

FIG. 8. Dependence of the pre-edge peak area in the $\mathrm{La} \mathrm{L}_{1}$-edge XANES spectrum on the $\mathrm{p}$ character ratio of the LDOS around the Fermi energy for the $\mathrm{La} \mathrm{L}_{3}$-edge of $\mathrm{La}_{4} \mathrm{PdO}_{7}, \mathrm{La}_{2} \mathrm{CuO}_{4}, \mathrm{LaCuSrO}_{4}, \mathrm{LaFeSrO}_{4}, \mathrm{LaCoO}_{3}$, and $\mathrm{LaAlO}_{3}$. Reprinted with permission from Asakura et al., Inorg. Chem. 53(12), 6048-6053 (2014). Copyright 2014 American Chemical Society.

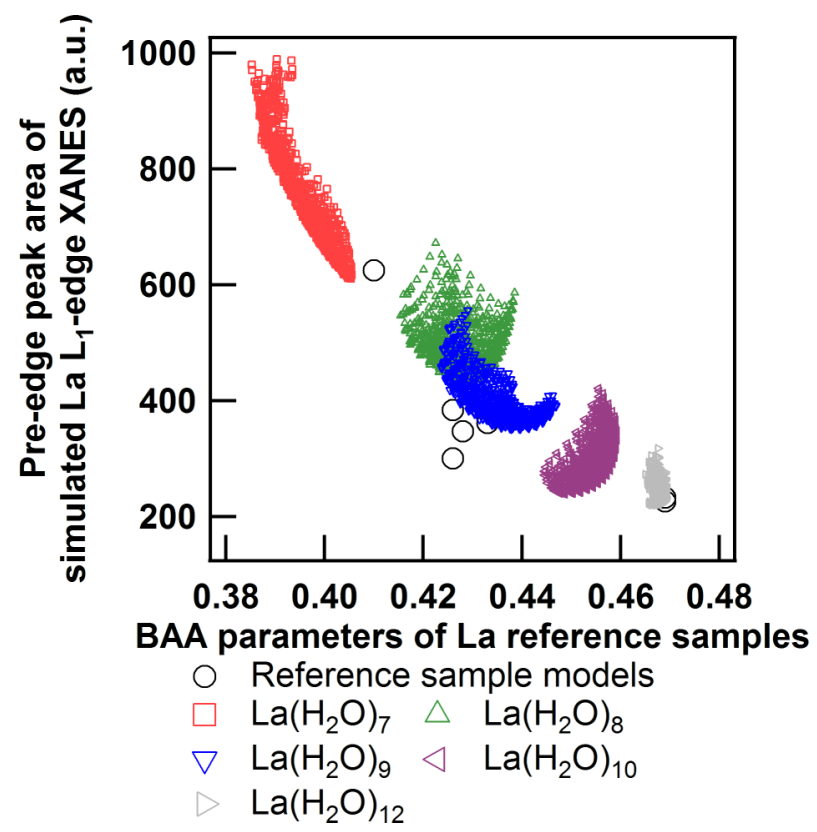

FIG. 9. Dependence of the peak area of the lowest energy peak of the simulated La $\mathrm{L}_{1}$-edge XANES spectrum on the BAA parameter for $\mathrm{La}$ aqueous complex models of $\mathrm{La}_{4} \mathrm{PdO}_{7}, \mathrm{La}_{2} \mathrm{CuO}_{4}, \mathrm{LaCuSrO}_{4}, \mathrm{LaFeSrO}_{4}$, $\mathrm{LaSrAlO}_{4}, \mathrm{LaCoO}_{3}$, and $\mathrm{LaAlO}_{3}$ and virtual complex models $\mathrm{La}\left(\mathrm{H}_{2} \mathrm{O}\right)_{n}$ $(n=7,8,9,10,12)$.

BAA parameter for the La complex models. It is noteworthy that the $\mathrm{La}\left(\mathrm{H}_{2} \mathrm{O}\right)_{7}$ models exhibit the widest distribution in Figure 9. This may indicate that for $\mathrm{La}\left(\mathrm{H}_{2} \mathrm{O}\right)_{7}$, the p contribution of La to the excited states can be easily changed by perturbation of the adjacent atoms. This is also important because most of the lanthanide trace elements in glasses are coordinated to 6-8 adjacent atoms, and Figure 9 indicates the possibility of distinguishing small structural changes in lanthanide dopants

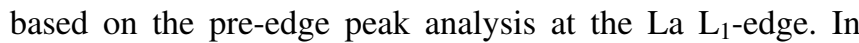
addition, we also performed theoretical simulation of the $\mathrm{La}$ $\mathrm{L}_{1}$-edge XANES spectrum of $\mathrm{La}\left(\mathrm{H}_{2} \mathrm{O}\right)_{6}\left(\right.$ Figure $\left.\mathrm{S} 7^{50}\right)$. In contrary to our expectation, the $\mathrm{La}\left(\mathrm{H}_{2} \mathrm{O}\right)_{6}$ model exhibits the larger pre-edge peak than $\mathrm{La}\left(\mathrm{H}_{2} \mathrm{O}\right)_{n}(n=7,8,9,10,12)$. We believe the discussion on the spectra of La compounds with $\geq 7$ adjacent oxygen atoms is fine. Further study on the pre-edge peak of $\mathrm{La}_{1}$-edge XANES of $\mathrm{La}\left(\mathrm{H}_{2} \mathrm{O}\right)_{m}(m=5,6,7)$ is undergoing.

\section{CONCLUSION}

This results of this study revealed that (1) DFT/ROCIS, TD-DFT, and FMS calculations can reproduce the dominant peaks of the $\mathrm{La} \mathrm{L}_{1}$-edge XANES of various La complex oxides using simple La aqueous complex models or La oxide cluster models. This means that these procedures are able to simulate the La $\mathrm{L}_{1}$-edge XANES spectra of La compounds with any structure at least in the absorption edge region. (2) The origin of the characteristic shoulder peak is the electric dipole transition from $2 \mathrm{~s}$ to hybridized states with $\mathrm{p}$ components. This has already been discussed in previous studies, but an interesting point is that the shoulder peak caused by the $p$ component contribution to unoccupied states can be an indicator of the geometric structure of La. Thus, the well-known pre-edge peak analysis of the K-edge XANES of 3d early transition metals, 
which is explained by the difference of the local structure of $3 \mathrm{~d}$ metals, can also be applied to the shoulder peak in the $\mathrm{L}_{1^{-}}$ edge XANES spectra of La. Unfortunately, it is still difficult to directly link the BAA parameter to physical properties. However, the present discussion with medium-scale theoretical simulations demonstrated that the BAA parameter can be an indicator of the local structure.

The present results might provide a way to analyze the local structure of each Ln element by L-edge XAS. We have already found a similar relationship to the relationship observed for La compounds between the XANES spectra and the local structure of other Ln elements, such as Pr, Nd, Sm, Gd, and Eu, which could be explained using the same strategy. In addition, the XANES analysis might give insight into a geometrical approach to investigate amorphous structures in combination with molecular dynamics, Monte Carlo simulations, and experimental data, such as pair distribution functions based on hard $\mathrm{X}$-ray or neutron diffraction.

\section{ACKNOWLEDGMENTS}

XAS measurements were performed under the approval of the Aichi Synchrotron Center, Aichi Science and Technology Foundation (Nos. 2501004 and 2503010). The authors thank Nagoya University Synchrotron Radiation Research Center for financial support for the XAS experiments. H.A. thanks Professor Tabuchi (Nagoya University Synchrotron Radiation Research Center) for discussions and suggestions. This research was partially supported by the Adaptable and Seamless Technology Transfer Program through Target-driven R\&D (A-STEP, No. AS242Z02825M) of the Japan Science and Technology Agency.

\section{NOMENCLATURE}

XAS $=$ X-ray absorption spectroscopy

EXAFS $=$ extended $X$-ray absorption fine structure

$\mathrm{XANES}=\mathrm{X}$-ray absorption near edge structure

${ }^{1}$ R. Yimnirun, J. Tangsritrakul, S. Rujirawat, and S. Limpijumnong, Ferroelectrics 381(1), 130-143 (2009).

${ }^{2}$ S. Suzuki, T. Takeda, A. Ando, and H. Takagi, Appl. Phys. Lett. 96(13), 132903 (2010).

${ }^{3}$ H. Ofuchi, J. Tsuchiya, N. Matsubara, M. Tabuchi, Y. Fujiwara, and Y. Takeda, Appl. Surf. Sci. 117-118, 781-784 (1997).

${ }^{4}$ H. Ofuchi, D. Kawamura, J. Tsuchiya, N. Matsubara, M. Tabuchi, Y. Fujiwara, and Y. Takeda, J. Synchrotron Radiat. 5(3), 1061-1063 (1998).

${ }^{5}$ M. A. Marcus and A. Polman, J. Non-Cryst. Solids 136(3), 260-265 (1991).

${ }^{6}$ M. R. Antonio, L. Soderholm, and A. J. G. Ellison, J. Alloys Compd. 250(1-2), 536-540 (1997).

${ }^{7}$ P. M. Peters and S. N. Houde-Walter, J. Non-Cryst. Solids 239(1-3), 162-169 (1998).

${ }^{8}$ G. Mountjoy, J. M. Cole, T. Brennan, R. J. Newport, G. A. Saunders, and G. W. Wallidge, J. Non-Cryst. Solids 279(1), 20-27 (2001).

${ }^{9}$ S. Kitano, N. Murakami, T. Ohno, Y. Mitani, Y. Nosaka, H. Asakura, K. Teramura, T. Tanaka, H. Tada, K. Hashimoto, and H. Kominami, J. Phys. Chem. C 117(21), 11008-11016 (2013).

${ }^{10}$ D. E. Sayers, E. A. Stern, and F. W. Lytle, Phys. Rev. Lett. 27(18), 1204-1207 (1971).

${ }^{11}$ E. M. Larson, A. J. G. Ellison, F. W. Lytle, A. Navrotsky, R. B. Greegor, and J. Wong, J. Non-Cryst. Solids 130(3), 260-272 (1991).

${ }^{12}$ I. Persson, E. D. Risberg, P. D'Angelo, S. De Panfilis, M. Sandström, and A. Abbasi, Inorg. Chem. 46(19), 7742-7748 (2007).

${ }^{13}$ P. D'Angelo, S. De Panfilis, A. Filipponi, and I. Persson, Chem. - Eur. J. 14(10), 3045-3055 (2008).
${ }^{14}$ I. Persson, P. D'Angelo, S. De Panfilis, M. Sandström, and L. Eriksson, Chem. - Eur. J. 14(10), 3056-3066 (2008).

${ }^{15}$ P. D’Angelo, A. Zitolo, V. Migliorati, G. Mancini, I. Persson, and G. Chillemi, Inorg. Chem. 48(21), 10239-10248 (2009).

${ }^{16} \mathrm{P}$. D'Angelo, A. Zitolo, V. Migliorati, and I. Persson, Chem. - Eur. J. 16(2), 684-692 (2010).

${ }^{17}$ D. Lundberg, I. Persson, L. Eriksson, P. D' Angelo, and S. De Panfilis, Inorg. Chem. 49(10), 4420-4432 (2010).

${ }^{18}$ P. D' Angelo, A. Zitolo, V. Migliorati, G. Chillemi, M. Duvail, P. Vitorge, S. Abadie, and R. Spezia, Inorg. Chem. 50(10), 4572-4579 (2011).

${ }^{19}$ P. D'Angelo, V. Migliorati, R. Spezia, S. De Panfilis, I. Persson, and A. Zitolo, Phys. Chem. Chem. Phys. 15(22), 8684-8691 (2013).

${ }^{20}$ P. D'Angelo and R. Spezia, Chem. - Eur. J. 18(36), 11162-11178 (2012).

${ }^{21}$ H. Asakura, T. Shishido, K. Teramura, and T. Tanaka, Inorg. Chem. 53(12), 6048-6053 (2014)

${ }^{22}$ H. Asakura, T. Shishido, S. Fuchi, K. Teramura, and T. Tanaka, J. Phys. Chem. C 118(36), 20881-20888 (2014).

${ }^{23} \mathrm{H}$. Asakura, T. Shishido, K. Teramura, and T. Tanaka, "Local Structure of $\mathrm{Eu}$, and Gd Complex Oxides and Their XANES Spectra" (unpublished).

${ }^{24}$ F. Neese, Wiley Interdiscip. Rev.: Comput. Mol. Sci. 2(1), 73-78 (2012).

${ }^{25}$ J. J. Rehr, J. J. Kas, M. P. Prange, A. P. Sorini, Y. Takimoto, and F. Vila, C. R. Phys. 10(6), 548-559 (2009).

${ }^{26}$ J. J. Rehr, J. J. Kas, F. D. Vila, M. P. Prange, and K. Jorissen, Phys. Chem. Chem. Phys. 12(21), 5503-5513 (2010).

${ }^{27}$ A. L. Ankudinov, C. E. Bouldin, J. J. Rehr, J. Sims, and H. Hung, Phys. Rev. B 65(10), 104107 (2002).

${ }^{28}$ S. G. Minasian, J. M. Keith, E. R. Batista, K. S. Boland, S. A. Kozimor, R. L. Martin, D. K. Shuh, T. Tyliszczak, and L. J. Vernon, J. Am. Chem. Soc. 135(39), 14731-14740 (2013).

${ }^{29}$ S. R. Daly, J. M. Keith, E. R. Batista, K. S. Boland, D. L. Clark, S. A. Kozimor, and R. L. Martin, J. Am. Chem. Soc. 134(35), 14408-14422 (2012).

${ }^{30}$ L. P. Spencer, P. Yang, S. G. Minasian, R. E. Jilek, E. R. Batista, K. S. Boland, J. M. Boncella, S. D. Conradson, D. L. Clark, T. W. Hayton, S. A. Kozimor, R. L. Martin, M. M. MacInnes, A. C. Olson, B. L. Scott, D. K. Shuh, and M. P. Wilkerson, J. Am. Chem. Soc. 135(6), 2279-2290 (2013).

${ }^{31}$ A. R. Jaszewski, S. Petrie, R. J. Pace, and R. Stranger, Chem. - Eur. J. 17(20), 5699-5713 (2011).

${ }^{32}$ M. Tabuchi, H. Asakura, N. Takao, H. Morimoto, N. Watanabe, Y. Baba, and Y. Takeda, "Hard X-ray XAFS beam-line, BL5S1, at AichiSR" (unpublished).

${ }^{33}$ B. Ravel and M. Newville, J. Synchrotron Radiat. 12(4), 537-541 (2005).

${ }^{34}$ D. Maganas, M. Roemelt, T. Weyhermuller, R. Blume, M. Havecker, A. Knop-Gericke, S. DeBeer, R. Schlogl, and F. Neese, Phys. Chem. Chem. Phys. 16(1), 264-276 (2014).

${ }^{35}$ D. Maganas, S. DeBeer, and F. Neese, Inorg. Chem. 53(13), 6374-6385 (2014).

${ }^{36}$ M. Roemelt, D. Maganas, S. DeBeer, and F. Neese, J. Chem. Phys. 138(20), 204101 (2013).

${ }^{37}$ D. Maganas, M. Roemelt, M. Havecker, A. Trunschke, A. Knop-Gericke, R. Schlogl, and F. Neese, Phys. Chem. Chem. Phys. 15(19), 7260-7276 (2013).

${ }^{38}$ F. Weigend and R. Ahlrichs, Phys. Chem. Chem. Phys. 7(18), 3297-3305 (2005).

${ }^{39}$ D. A. Pantazis and F. Neese, J. Chem. Theory Comput. 5(9), 2229-2238 (2009).

${ }^{40}$ C. van Wüllen, J. Chem. Phys. 109(2), 392-399 (1998).

${ }^{41}$ A. K. Rappe, C. J. Casewit, K. S. Colwell, W. A. Goddard, and W. M. Skiff, J. Am. Chem. Soc. 114(25), 10024-10035 (1992).

${ }^{42}$ M. Hanwell, D. Curtis, D. Lonie, T. Vandermeersch, E. Zurek, and G. Hutchison, J. Cheminf. 4(1), 17 (2012).

${ }^{43}$ B. Ravel, J. Synchrotron Radiat. 8(2), 314-316 (2001).

${ }^{44}$ G. J. Ackland and A. P. Jones, Phys. Rev. B 73(5), 054104 (2006).

${ }^{45}$ C. Brouder, J. Phys.: Condens. Matter 2(3), 701 (1990).

${ }^{46}$ G. Materlik, B. Sonntag, and M. Tausch, Phys. Rev. Lett. 51(14), 1300-1303 (1983).

${ }^{47}$ J. Chaboy, J. García, and A. Marcelli, J. Magn. Magn. Mater. 166(1-2), 149164 (1997).

${ }^{48}$ N. Ishimatsu, R. Sasada, H. Maruyama, T. Ichikawa, H. Miyaoka, T. Kimura, M. Tsubota, Y. Kojima, T. Tsumuraya, T. Oguchi, N. Kawamura, and A. Machida, J. Phys.: Conf. Ser. 190(1), 012070 (2009).

${ }^{49}$ S. DeBeer George, P. Brant, and E. I. Solomon, J. Am. Chem. Soc. 127(2), 667-674 (2004).

${ }^{50}$ See supplementary material at http://dx.doi.org/10.1063/1.4919055 for TD-DFT theoretical simulations of La L1-edge XANES spectra of La aqueous complex models. 